\title{
Baicalein, a Natural Anti-Cancer Compound, Alters MicroRNA Expression Profiles in Bel-7402 Human Hepatocellular Carcinoma Cells
}

\author{
Beibei Bie ${ }^{a, b}$ Jin Sun ${ }^{a, b}$ Jun Li $i^{a, b}$ Ying Guo ${ }^{a, b}$ Wei Jiang ${ }^{a, b} \quad$ Chen Huang ${ }^{b, c}$ \\ Jun Yang ${ }^{a, b}$ Zongfang $L^{a}{ }^{a, b}, c$ \\ aNational \& Local Joint Engineering Research Center of Biodiagnosis and Biotherapy, the second \\ affiliated hospital, Xi' an Jiaotong University, Xi'an, 'bShaanxi Provincial Clinical Research Center for \\ Hepatic \& Splenic Diseases, Shaanxi, Xi'an, 'Key Laboratory of Environment and Disease-Related Gene, \\ Ministry of Education, Xi'an Jiaotong University, Xi'an, China
}

\section{Key Words}

Baicalein • Hepatocellular carcinoma $•$ Anti-proliferative $\cdot$ MicroRNA $\bullet$ Expression profiling

\begin{abstract}
Background/Aims: Baicalein has been shown to possess significant anti-hepatoma activity by inhibiting cell proliferation. Whether the anti-proliferative effect of baicalein is related to its modulation of miRNA expression in hepatocellular carcinoma (HCC) is still unknown. Methods: The anti-proliferative effects of baicalein on HCC cell line Bel-7402 was assessed by detecting the proliferation activity, cell cycle distribution, expression changes of p21/CDKN1A, P27/ CDKN1B, total Akt and phosphoryted AKT. Microarray analysis was conducted to determine the miRNA expression profiles in baicalein-treated or untreated Bel-7402 cells and then validated by qRT-PCR in two HCC cell lines (Bel-7402 and Hep3B). The gain-of-function of miR-3127-5p was performed by detecting anti-proliferative effects after transfecting miRNA mimics in cells. Finally, the expression level of miR-3127-5p in different HCC cell lines was determined by qRT-PCR. Results: Baicalein was able to inhibit the proliferation of Bel-7402 cells by inducing cell cycle arrest at the $S$ and G2/M phase via up-regulating the expression of p21/CDKN1A and P27/CDKN1B and suppressing the PI3K/Akt pathway. Baicalein could alter the miRNA expression profiles in Bel-7402 cells. Putative target genes for differentially expressed miRNAs could be enriched in terms of cell proliferation regulation, cell cycle arrest and were mainly involved in MAPK, PI3K-Akt, Wnt, Hippo and mTOR signaling pathways. MiR3127-5p, one of up-regulated miRNAs, exhibits low expression level in several HCC cell lines and its overexpression could inhibit cell growth of Bel-7402 and Hep3B cell lines by inducing $S$ phase arrest by up-regulating the expression of p21and P27 and repressing the PI3K/Akt pathway. Conclusions: Modulation of miRNA expression may be an important mechanism underlying the anti-hepatoma effects of baicalein.




\section{Cellular Physiology Cell Physiol Biochem 2017;41:1519-1531 \\ \begin{tabular}{l|l} 
and Biochemistry Published onIIne: VIarch 24, 2017 & $\begin{array}{l}\text { (c) 2017 The Author(s). Published by S. Karger AG, Basel } \\
\text { www.karger.com/cpb }\end{array}$ \\
\hline
\end{tabular} \\ Bie et al.: Baicalein Alters miRNA Expression Profiles in Bel-7402 Liver Cancer Cells}

\section{Introduction}

Hepatocellular carcinoma (HCC) is the sixth most common malignancy and the third principal cause of cancer deaths worldwide due to its very poor prognosis and the lack of curative treatment options after diagnosis, especially in, East and Southeast Asia, Africa and Southern Europe [1].

Chinese herbal medicines have been widely used for HCC prevention and treatment as a noticeable choice for its multi-level, multi-target and coordinated intervention effects and their significant anti-cancer effects against HCC have been identified, such as curcumin, baicalein, resveratrol etc., indicating that Chinese herbal compounds represent an important medical and pharmaceutical resource for the development of new treatments for HCC [2,3].

Baicalein (5, 6, 7-trihydroxyflavone, Fig. 1), a purified flavonoid compound with defined chemical structure extracted from the dry roots of Scutellaria radix that is a broadly used herb in China, has been demonstrated that it has potential anti-tumor effects on many types of cancer cells, including HCC [4]. Previous studies have demonstrated that baicalein can inhibit the growth, invasion and metastasis of HCC through inducing cell cycle arrest and apoptosis, autophagy via several cancer-related signaling or molecules including MEK/ ERK, JNK, Bcl-2, mTOR, CD24, 12-LOX, NF-кB [5-11]. MicroRNAs (miRNAs) are a large class of short non-coding RNAs, which paly critical roles in human carcinogenesis by mediating the post-transcriptional regulation of protein-coding genes, including HCC $[12,13]$, and accumulating evidence indicates that miRNAs may act as oncogenes or tumor suppressor genes and appear to be markedly diagnostic biomarkers and prognostic factors that exhibits strong potential as therapeutic molecules and targets for HCC treatment [14]. In recent years, emerging studies have demonstrated that Chinese medicinal herbs can exert their anti-tumor effects through targeting miRNAs [15]. For example, curcumin could alter the miRNA expression profile in human retinoblastoma and pancreatic cancer cells, involving some well-known cancer-related miRNAs, such as miR-22, a tumor-suppressor miRNA was upregulated after curcumin treatment $[16,17]$. Curcumin also can promote the apoptosis of cancer cells via miR-186, miR-15a and miR-16 and can inhibit the proliferation, invasion and metastasis of cells via miR-21, miR-208 [18-21]. Camptothecin is able to induce the apoptosis of cancer cells via microRNA-125b-mediated mitochondrial pathways and also can induce autophagy of cancer cells and enhance its chemosensitivity by miR-15a and miR-16 [22, 23]. A recent study found that andrographolide could altered miRNA expression profile and its downstream signals in hepatoma tumor tissues from mice [24]. Additionally, another recent study showed that resveratrol could regulate apoptotic and cell cycle machinery in breast cancer cells by modulating key tumor-suppressive miRNAs including miR-125b-5p, miR200c-3p, miR-409-3p, miR-122-5p and miR-542-3p [25]. However, there is still no related report about the effects of baicalein on miRNA expression profile when it exerts its inhibition on hepatoma tumor growth.

Based on the above information, we hypothesize that baicalein can inhibit HCC growth by affecting miRNA expression. In the present study, we first evaluated the anti-hepatoma effects of Bel-7402 that a HCC cell lines and detected the changes of miRNA expression after the Bel-7402 cells were treated or untreated with baicalein using miRNA microarray, then validated the differentially expressed miRNAs in two HCC cell lines and identified the antihepatoma effects of mir-3127-5p that a baicalein up-regulated miRNA in vitro. Our findings suggest that modulation of miRNA expression may be an important mechanism underlying the anti-hepatoma effects of baicalein and lay the groundwork for further investigations.

\section{Materials and Methods}

Cell culture and baicalein treatments

Human HCC cell lines Bel-7402, Bel-7404, Hep3B, HepG2, Huh-7, MHCC97H, SMMC-7721 and the normal hepatic cell line L-O2 were purchased from the Cell Bank of Type Culture Collection of the Chinese 


\section{Cellular Physiology Cell Physiol Biochem 2017;41:1519-1531

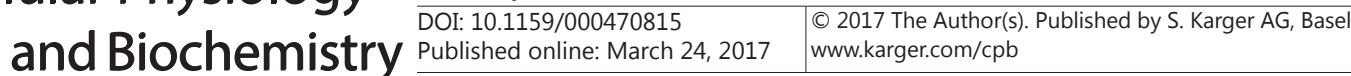

Fig. 1. Chemical structure of baicalein. The molecular formula of baicalein is $\mathrm{C}_{15} \mathrm{H}_{10} \mathrm{O}_{5}$.

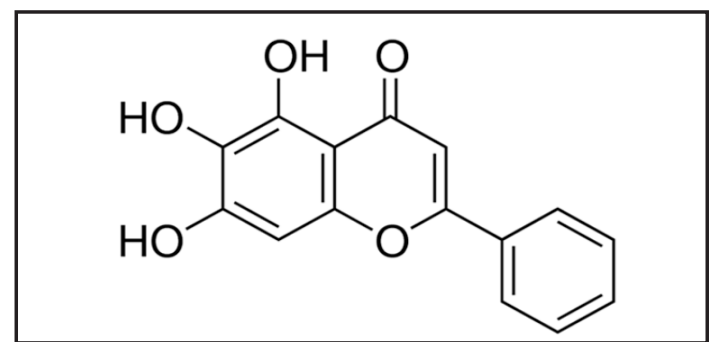

Academy of Sciences (Shanghai, China). Baicalein was purchased from Sigma-Aldrich (465119, USA). All cell lines were cultured in Dulbecco's modified Eagle's medium (Hyclone, USA) supplemented with 10\% fetal bovine serum (Gibco, USA), 1\% penicillin-streptomycin $(100 \mathrm{U} / \mathrm{ml}$ penicillin and $100 \mu \mathrm{g} / \mathrm{ml} \mathrm{streptomycin;}$ Hyclone, USA) at $37^{\circ} \mathrm{C}$ in a humidified atmosphere with $5 \% \mathrm{CO}$. For baicalein treatment, the cells were seeded in plate with appropriately concentration and incubated overnight, then treated with various concentration $(0,40$ and $80 \mu \mathrm{M})$ of baicalein dissolved in DMSO (final DMSO concentration in growth media, $0.1 \%)$.

\section{CCK-8 for Cell viability assay}

Cell viability was assessed with CCK-8. Briefly, Cells were seeded in 96-well plate at 4000 cells per well and incubated overnight. Then treated for $24 \mathrm{~h}$ or $48 \mathrm{~h}$ with a different concentration of baicalein or transfected with miRNA mimics and negative control (NC). At the indicated times, CCK-8 was added and incubated for $1 \mathrm{~h}$ at $37^{\circ} \mathrm{C}$. The optical density (OD) of each well at $450 \mathrm{~nm}$ was detected by microplate reader (TECAN infinite M20 PRO, Switzerland). Relative cell viability was calculated with the following formula: relative cell viability $(\%)=$ OD (treatment group) $/$ OD (control group) $\times 100 \%$.

\section{Cell cycle analysis}

The cells were harvested and fixed with ice-cold $80 \%$ ethanol overnight at $4^{\circ} \mathrm{C}$. After washing with PBS, the cells were treated with propidium iodide (PI) staining solution containing $50 \mu \mathrm{g} / \mathrm{ml} \mathrm{PI,} 100 \mu \mathrm{g} / \mathrm{ml}$ RNase A and $0.2 \%(\mathrm{v} / \mathrm{v}$ ) Triton X-100 at room temperature for $30 \mathrm{~min}$ in the dark. The percentage of cells in various phases of the cell cycle was determined by a flow cytometer (BD, USA) with FlowJo software (Tree star, USA).

\section{RNA preparation and miRNAs microarray assay}

After $24 \mathrm{~h}$ incubation with baicalein, the cells were harvested and the total RNA including miRNAs was extracted using TRIzol reagent (Invitrogen, USA) according to the manufacturers' instructions. The concentration and quality of the RNA were assessed by Nanodrop 2000 spectrophotometry (Thermo Fisher Scientific, USA) and formaldehyde denaturing gel electrophoresis.

$1 \mu \mathrm{g}$ of total RNA was labeled with biotin using FlashTag® Biotin HSR RNA Labeling Kit (Genisphere, USA) according to the manufacturer's instructions and subsequently hybridized to Affymetrix GeneChip $®$ miRNA 4.0 Arrays, containing 2, 578 mature human miRNAs, at $48{ }^{\circ} \mathrm{C}$ and $60 \mathrm{rpm}$ for $16 \mathrm{~h}$. The arrays were washed and stained using the GeneChip ${ }^{\circledR}$ Hybridization Wash and Stain Kit in the 450 Fluidics Station and were then scanned with the GeneChip Scanner 3000 7G (Affymetrix, USA) to acquire fluorescent images of each array. The raw data (CEL files) were extracted from the scanned images using the Affymetrix GeneChip Command Console and were then analyzed with Affymetrix Expression Console software using the Affymetrix default analysis settings and quantile as the normalization method. Raw and processed microarray data have been deposited in Gene Expression Omnibus (GEO) at NCBI (http://www.ncbi.nlm. nih.gov/geo/) with accession number GSE85511.

\section{Data analysis of miRNAs microarray}

The raw data (CEL files) were uploaded to the online working platform of Gene-Cloud Biotechnology Information (GCBI) (https://www.gcbi.com.cn/gclab/html/index, Gminix Informatics Co., Ltd., Shanghai, China) for further difference analysis of miRNAs profiles. A relative fold change $>1.5$ in the differential expression of miRNAs and a p value $<0.05$ were considered significant. The Gene Cluster (version 3.0) and Java TreeView software programs were used to perform the hierarchical cluster analysis of differentially expressed miRNAs and to visualize the miRNAs. 


\section{Cellular Physiology Cell Physiol Biochem 2017;41:1519-1531 \begin{tabular}{ll|l} 
and Biochemistry 10.1159/000470815 & $\begin{array}{l}\text { Published onlIne: } 2017 \text { Tharch 24, Author(s). Published by S. Karger AG, Basel } \\
\text { www.karger.com/cpb }\end{array}$ \\
\hline
\end{tabular}

Target prediction and GO and KEGG pathway analysis

The target genes of miRNA were predicted by the overlapped data from miRanda (http:// www. microrna.org/microrna/home.do) and TargetScan prediction software (http://www.targetscan.org/). The predicted target genes were annotated with Gene Ontology (GO) and Kyoto Encyclopedia Genes \& Genomes (KEGG) pathways using the DAVID gene annotation tool (https://david.ncifcrf.gov/) and statistically over-represented GO terms in the biological process and KEGG pathway were obtained by applying a Fisher's exact $p$-value cutoff $<0.01$ and correcting for multiple testing with the Benjamini false discovery rate (FDR).

\section{Quantitative real-time PCR}

For miRNA analysis, the first-strand cDNA was synthesized using the Mir-X ${ }^{\mathrm{TM}}$ miRNA First-Strand Synthesis Kit (TaKaRa, Japan). qRT-PCR was performed using SYBR Premix Ex Taq II (TaKaRa, Japan) on an ABI 7500 fast real-time PCR system (Applied Biosystems, USA) and using U6 snRNA as endogenous control.

For mRNA analysis, the first-strand cDNA was synthesized using the Primescript ${ }^{\circledR}$ RT reagent kit with gDNA Eraser (TaKaRa, Japan). qRT-PCR was performed using SYBR Select Master Mix (Applied Biosystems, USA) on an ABI 7500 fast real-time PCR system (Applied Biosystems, USA) and using beta-actin as endogenous control.

A dissociation curve was performed to ensure the validity of each specific PCR product. The experiments were independently performed three times in triplicate. All samples were normalized to endogenous control and determined using the $2^{-\Delta \Delta \mathrm{Ct}}$-analysis methods. The primers that were used in the qRT-PCR are listed in Table 1.

\section{Western blotting}

The harvested cells were lysed with M-PER ${ }^{\mathrm{TM}}$ Mammalian Protein Extraction Reagent (Thermo Fisher Scientific, USA) that containing Pierce Protease and Phosphatase Inhibitor Tablet (Thermo Fisher Scientific, USA). Then, samples (20 $\mu$ g protein/lane) were electrophoresed by SDS-polyacrylamide gel electrophoresis (PAGE) and transferred onto PVDF membrane. The membrane was blocked with Western Blocking Buffer (CWBIO, China) for $1 \mathrm{~h}$ at room temperature and subsequently incubated overnight at $4^{\circ} \mathrm{C}$ with anti-p21/ CDKN1A (1:1000; CST, \#2947), anti-p27/CDKN1B (1:1000; CST, \#3686), anti-Akt (1:1000; CST, \#4691), anti-p-Akt (Thr308) (1:1000; CST, \#13038), anti-p-Akt (Ser473) (1:1000; CST, \#4060) and anti- $\beta$-actin (1:1000; CST, \#4970). Then, the membrane was incubated with a horseradish peroxidase-conjugated goatanti-rabbit IgG (1:10000; Millipore区\#AP307P, USA) for $1 \mathrm{~h}$. Chemiluminescence was detected using the SuperSignal West pico chemiluminescent (Thermo Fisher Scientific, USA).

\section{MiRNA mimics transfection}

Bel7-402 and Hep3B cells were seeded in plates and grown to 30-50\% confluency before transfection. The cells were then transfected with miR-3127-5p mimics (Invitrogen, USA) or negative control (NC) mimics (Invitrogen, USA) a final concentration of $100 \mathrm{nM}$ using Lipofectamine RNAi max (Invitrogen, USA) following the manufacturer's protocol. The miR-3127-5p levels were determined by qRT-PCR 24-48 h after transfection.

\section{Statistical analysis}

Each experiment was repeated three biological replicates. All of the statistical evaluations were performed using the SPSS (version 20) software package. The results are presented as the mean \pm standard deviation (SD). The differences between the two groups and multiple groups were determined by Student's t-test and one-way analysis of variance (ANOVA), respectively. $p<0.05$ was considered statistically significant. 


\section{Cellular Physiology Cell Physiol Biochem 2017;41:1519-1531

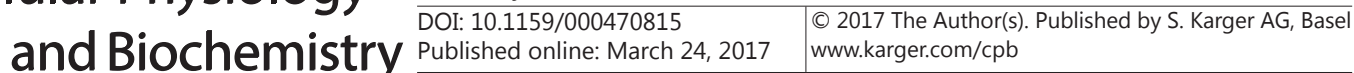 \\ Bie et al.: Baicalein Alters miRNA Expression Profiles in Bel-7402 Liver Cancer Cells}

\section{Results}

Baicalein inhibits the proliferation of HCC cell line Bel-7402 by inducing cell cycle arrest at the $S$ and G2/M phase via up-regulating the expression of $21 / C D K N 1 A$ and P27/CDKN1B and suppressing the PI3K/Akt pathway

To evaluate anti-tumor effects of baicalein on bel-7402 cells, a HCC cell line that endogenously expresses wild type p53, we compared the cytotoxic activity of baicalein on HCC cell line Bel-7402 and normal liver cell line L-O2 in vitro. The anti-proliferation effects of baicalein were examined by CCK-8 assay after treatment with $0,20,40,60,80$ and 100 $\mu \mathrm{M}$ of baicalein for 24 and $48 \mathrm{~h}$. As shown in Fig. 2A, the viability of Bel-7402 cells was both significantly reduced by baicalein in dose-dependent manner after treatment for $24 \mathrm{~h}$ and $48 \mathrm{~h}$, whereas the normal liver cell line L-O2 was almost unaffected. Cellular proliferation inhibition rate was about $70 \%$ after treating with $80 \mu \mathrm{M}$ baicalein for $24 \mathrm{~h}$ and could reach up to about $80 \%$ after treating for $48 \mathrm{~h}$.

To further investigate the mechanism by which baicalein induces anti-proliferation in Bel-7402 cells, cell cycle analysis was performed on cells treated with 0,40 and $80 \mu \mathrm{M}$ baicalein for $24 \mathrm{~h}$, we found that a significant increase in the number of $\mathrm{S}$ and G2/M-phase cells, whereas a decrease in the percentage of cells in G0/G1-phase were observed compared to $0 \mu \mathrm{M}$ (DMSO control) treatment group, indicating baicalein arrested the cell cycle of Bel7402 cells at $\mathrm{S}$ and G2/M phase. (Fig. 2B and C). Furthermore, qRT-PCR and western blot results indicated that both mRNA and protein level of p21/CDKN1A and P27/CDKN1B, two key cell-cycle inhibitors contribute to $S$ and G2/M phase arrest, was significantly upregulated by baicalein (Fig. 2D, E and F).

Previous studies demonstrated that baicalein could exert its anti-tumor effect through modulating the PI3K/AKT signaling pathway in several cancer types [26-28]. To explore the effect of baicalein on PI3K/AKT pathway, the phosphorylation level of AKT at Thr308 and Ser473 in Bel-7402 cells treated with 0,40 and $80 \mu \mathrm{M}$ baicalein for $24 \mathrm{~h}$ was detected by western blotting, we found the phosphorylation level of AKT at both Thr308 and Ser473 were significantly decreased by baicalein treatment (Fig. 2G and $\mathrm{H}$ ), indicating baicalein could suppress the PI3K/AKT pathway.

Baicalein treatment alters miRNA expression profiles of Bel-7402 cells

To study the responses of miRNAs to baicalein, we profiled the miRNA expression in HCC cell line Bel-7402 treated with different concentrations of baicalein $(0,40$ and $80 \mu \mathrm{M})$ for 24 hours using microarray. We found 43 miRNAs with a more than 1.5 -fold change were both significantly differentially expressed in $40 \mu \mathrm{M}$ and $80 \mu \mathrm{M}$ baicalein treated cells compared to its DMSO control $(\mathrm{p}<0.05)$, of which 13 were up-regulated and 30 were down-regulated (Fig. 3 and Table 2).

Validation of miRNA microarray results by qRT-PCR

To validate the results obtained by the miRNA microarray, the expression of six differentially expressed miRNAs were detected by qRT-PCR in two HCC cell lines Bel-7402 and Hep3B. Consistent with the results from the miRNA microarrays, miR-1972, miR3127-5p, miR-3663-3p and miR-25-5p were up-regulated in the HCC cell line Bel-7402 and Hep3B, whereas miR-4286 and miR-675-5p were down-regulated (Fig. 4). Thus, qRT-PCR results showed that expression patterns of these six selected miRNAs were concordant with microarray and demonstrated that the microarray data were reliable.

\section{Target prediction of differentially expressed miRNAs and its functional analysis}

In order to gain insight into the probable biological function of the differentially expressed miRNAs, we first predicted the putative targets of all 43 baicalein-regulated miRNAs by the overlapped data from miRanda and TargetScan prediction software. As shown in online suppl. material, Table S1 (see www.karger.com/doi/10.1159/000470815) there are total 4,356 mRNA transcripts were identified as putative target of 43 differentially expressed 
A
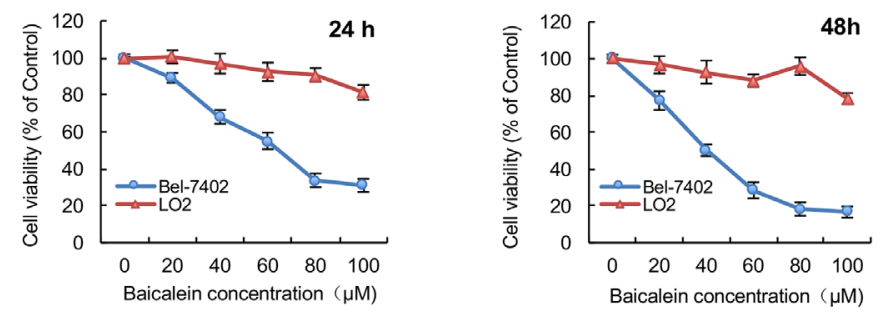

B

C
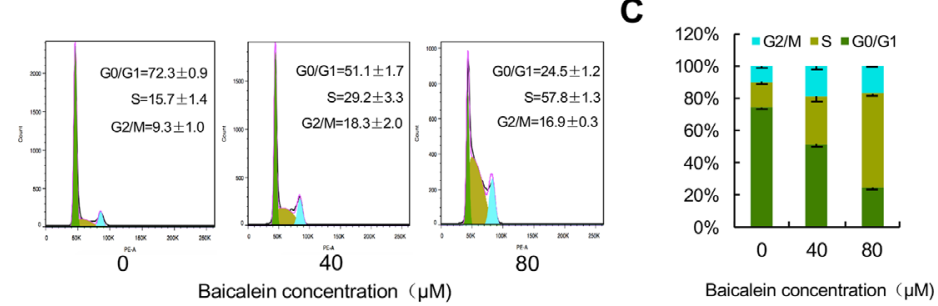

D

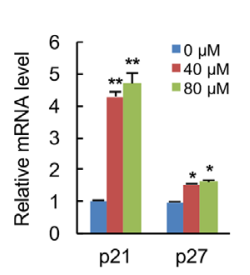

E

Baicalein concentration $(\mu \mathrm{M})$

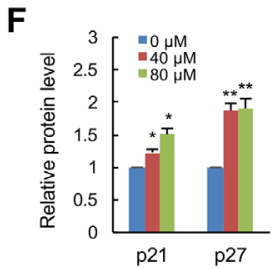

G

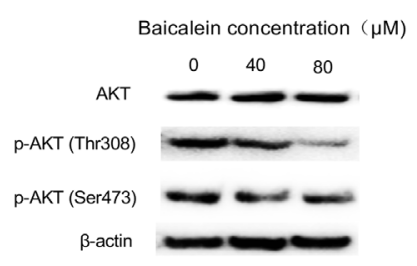

H

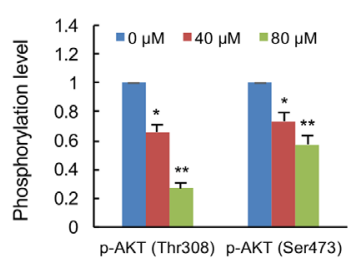

Fig. 2. Baicalein inhibits the proliferation of HCC cell line Bel-7402 by inducing cell cycle arrest at the $S$ phase. (A) Analysis of cell proliferation using CCK-8 assay, Bel-7402 cells were incubated with baicalein (0-100 $\mu \mathrm{M}$ ) for $24 \mathrm{~h}$ or $48 \mathrm{~h}$; The cell cycle distribution is shown by flow cytometric histogram (B) and a stacked column chart (C), Bel-7402 cells were treated with baicalein for $24 \mathrm{~h}$; mRNA level (D) and protein level (E, western bolt image; F, quantitative results) of p21 and p27 in Bel-7402 cells treated with baicalein were determined by qRT-PCR and western blotting, respectively; protein level (G, western bolt image; $\mathrm{H}$, quantitative results) of total Akt, p-Akt (Thr 308) and p-Akt (Ser473) in Bel-7402 cells treated with baicalein were assessed by western blotting. Data are shown as the mean \pm SD based on three independent experiments. ${ }^{*} p<0.05,{ }^{* *} p<0.01$.

miRNAs. Subsequencely, the basic biological function of putative target gene was classified by Gene Ontology (GO) and KEGG pathway analysis. As shown in Fig. 5, Gene ontology (GO) analysis indicated that target genes of 43 differentially expressed miRNAs could be enriched in terms of regulation of cell proliferation, cell cycle, cell cycle arrest, apoptosis process, cell migration etc. (Fig. 5A). KEGG pathway analysis showed that miRNA target genes were mainly involved in MAPK, PI3K-Akt, Wnt, Hippo, and mTOR signaling pathway (Fig. 5B).

MiR-3127-5p, a miRNA up-regulated by baicalein, its overexpression suppresses the proliferation of Bel-7402 cells and exhibits a lower expression in HCC cells

To investigate the functional role of baicalein-regulated miRNA in HCC cells, we overexpressed the miR-3127-5p that a miRNA up-regulated by baicalein in HCC cell line Bel- 


\section{Cellular Physiology and Biochemistry

Table 2. Differentially expressed miRNAs between DMSO control and baicalein treated Bel-7402 cell (Fold change $>1.5 ; p<0.05$ )

\begin{tabular}{|c|c|c|c|c|c|c|}
\hline miRNA Name & Accession ID & $\begin{array}{l}\text { Fold change } \\
\text { (0 vs } 40 \text { ) }\end{array}$ & $\begin{array}{l}\text { p value } \\
(0 \text { vs } 40)\end{array}$ & $\begin{array}{l}\text { Fold change } \\
(0 \text { vs } 80)\end{array}$ & $\begin{array}{l}p \text { value } \\
(0 \text { vs } 80)\end{array}$ & Regulation \\
\hline hsa-let-7e-3p & MIMAT0004485 & -1.97 & 0.015 & -2.51 & 0.011 & down \\
\hline hsa-miR-19a-3p & MIMAT0000073 & -1.63 & 0.027 & -1.67 & 0.033 & down \\
\hline hsa-miR-7-5p & MIMAT0000252 & -2.88 & 0.011 & -4.18 & 0.023 & down \\
\hline hsa-miR-222-5p & MIMAT0004569 & -2.04 & 0.019 & -4.00 & 0.002 & down \\
\hline hsa-miR-23b-5p & MIMAT0004587 & -1.90 & 0.003 & -1.84 & 0.007 & down \\
\hline hsa-miR-191-3p & MIMAT0001618 & -1.72 & 0.009 & -1.94 & 0.009 & down \\
\hline hsa-miR-186-5p & MIMAT0000456 & -1.57 & 0.016 & -1.57 & 0.018 & down \\
\hline hsa-miR-30e-3p & MIMAT0000693 & -1.62 & 0.025 & -1.95 & 0.001 & down \\
\hline hsa-miR-452-5p & MIMAT0001635 & -1.60 & 0.014 & -1.95 & 0.003 & down \\
\hline hsa-miR-675-5p & MIMAT0004284 & -2.05 & 0.004 & -2.10 & 0.006 & down \\
\hline hsa-miR-675-3p & MIMAT0006790 & -2.33 & 0.009 & -2.73 & 0.010 & down \\
\hline hsa-miR-1303 & MIMAT0005891 & -2.41 & 0.006 & -1.74 & 0.031 & down \\
\hline hsa-miR-3136-5p & MIMAT0015003 & -1.69 & 0.011 & -1.53 & 0.023 & down \\
\hline hsa-miR-4284 & MIMAT0016915 & -1.65 & 0.020 & -1.80 & 0.008 & down \\
\hline hsa-miR-4286 & MIMAT0016916 & -3.11 & 0.003 & -3.36 & 0.004 & down \\
\hline hsa-miR-4461 & MIMAT0018983 & -2.46 & 0.026 & -2.90 & 0.002 & down \\
\hline hsa-miR-4472 & MIMAT0018999 & -3.40 & 0.010 & -3.28 & 0.015 & down \\
\hline hsa-miR-4710 & MIMAT0019815 & -1.58 & 0.042 & -2.20 & 0.026 & down \\
\hline hsa-miR-4720-5p & MIMAT0019833 & -2.97 & 0.024 & -4.56 & 0.008 & down \\
\hline hsa-miR-5680 & MIMAT0022468 & -2.98 & 0.030 & -9.33 & 0.002 & down \\
\hline hsa-miR-6735-5p & MIMAT0027371 & -2.66 & 0.023 & -3.07 & 0.016 & down \\
\hline hsa-miR-6742-5p & MIMAT0027385 & -1.71 & 0.032 & -1.87 & 0.024 & down \\
\hline hsa-miR-6754-5p & MIMAT0027408 & -2.22 & 0.005 & -2.07 & 0.015 & down \\
\hline hsa-miR-6757-5p & MIMAT0027414 & -1.66 & 0.026 & -1.62 & 0.011 & down \\
\hline hsa-miR-6774-5p & MIMAT0027448 & -2.15 & 0.010 & -2.49 & 0.003 & down \\
\hline hsa-miR-5194 & MIMAT0021125 & -2.18 & 0.025 & -3.30 & 0.013 & down \\
\hline hsa-miR-1304-5p & MIMAT0005892 & -2.02 & 0.011 & -2.18 & 0.021 & down \\
\hline hsa-miR-3194-5p & MIMAT0015078 & -1.76 & 0.006 & -2.00 & 0.006 & down \\
\hline hsa-miR-1247-5p & MIMAT0005899 & -2.72 & 0.007 & -2.65 & 0.006 & down \\
\hline hsa-miR-18b-5p & MIMAT0001412 & -1.63 & 0.033 & -1.70 & 0.023 & down \\
\hline hsa-miR-25-5p & MIMAT0004498 & 1.75 & 0.015 & 1.95 & 0.006 & up \\
\hline hsa-miR-424-3p & MIMAT0004749 & 1.67 & 0.009 & 1.54 & 0.030 & up \\
\hline hsa-miR-1972 & MIMAT0009447 & 2.66 & 0.003 & 4.20 & 0.001 & up \\
\hline hsa-miR-3127-5p & MIMAT0014990 & 2.38 & 0.001 & 2.74 & 0.014 & up \\
\hline hsa-miR-3178 & MIMAT0015055 & 2.57 & 0.005 & 2.51 & 0.006 & up \\
\hline hsa-miR-3195 & MIMAT0015079 & 3.25 & 0.003 & 2.92 & 0.009 & up \\
\hline hsa-miR-3663-3p & MIMAT0018085 & 3.40 & 0.006 & 4.07 & 0.020 & up \\
\hline hsa-miR-4443 & MIMAT0018961 & 2.54 & 0.004 & 2.73 & 0.004 & up \\
\hline hsa-miR-4497 & MIMAT0019032 & 2.44 & 0.017 & 2.09 & 0.025 & up \\
\hline hsa-miR-4516 & MIMAT0019053 & 1.73 & 0.007 & 1.93 & 0.002 & up \\
\hline hsa-miR-4674 & MIMAT0019756 & 2.27 & 0.008 & 1.96 & 0.039 & up \\
\hline hsa-miR-5100 & MIMAT0022259 & 1.79 & 0.013 & 1.88 & 0.014 & up \\
\hline hsa-miR-6807-5p & MIMAT0027514 & 2.58 & 0.002 & 4.05 & 0.004 & up \\
\hline
\end{tabular}

Fig. 3. Hierarchical clustering of miRNAs differentially expressed in baicalein treated or untreated Bel-7402 cells. A hierarchical clustered heat map showing the $\log 2$ transformed expression values for differentially expressed miRNAs (Fold-change $\geq 1.5 ; p \leq 0.05$ ) between baicalein treated (40 and $80 \mu \mathrm{M}$ ) or untreated (DMSO control) Bel-7402 cells. The intensity of the color scheme is calibrated to the $\log 2$ expression values such that red refers to higher transcript abundance and green refers to lower transcript abundance. The bar code on the right represents the color scale of the $\log 2$ values. Each column represents the data from one of three biological replicates of each sample.

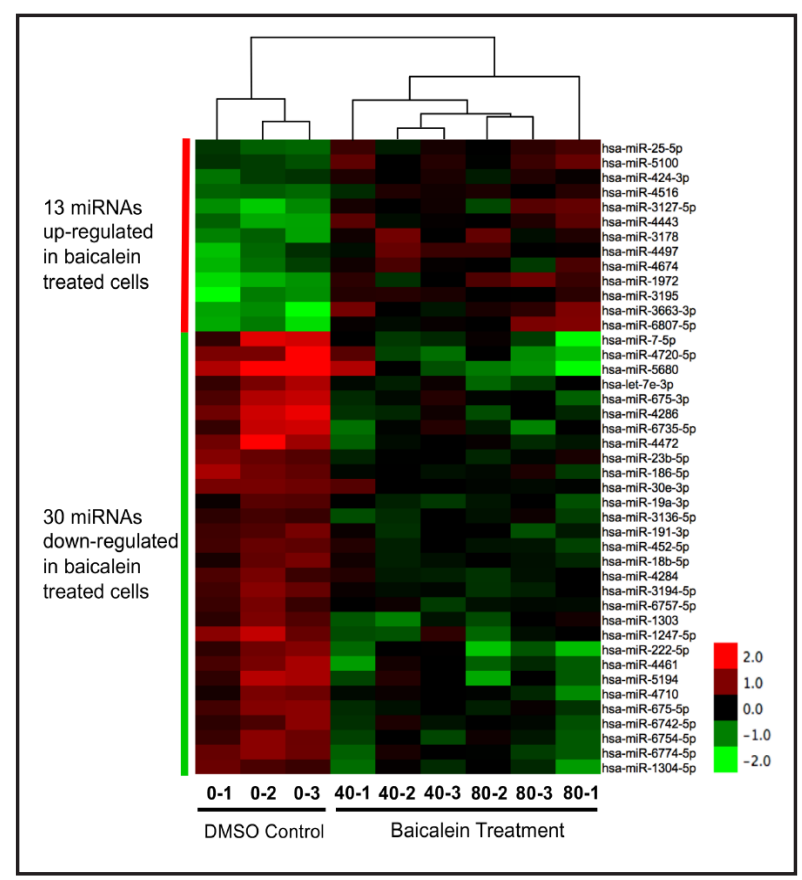

7402 and Hep3B by transfecting miR-3127-5p or negative control (NC) mimics. As shown in Fig. 6A, the overexpression of miR-3127-5p could significantly inhibit cell growth of Bel7402 and Hep3B cell lines, whereas the NC mimics had no effect. Cell cycle analysis showed 


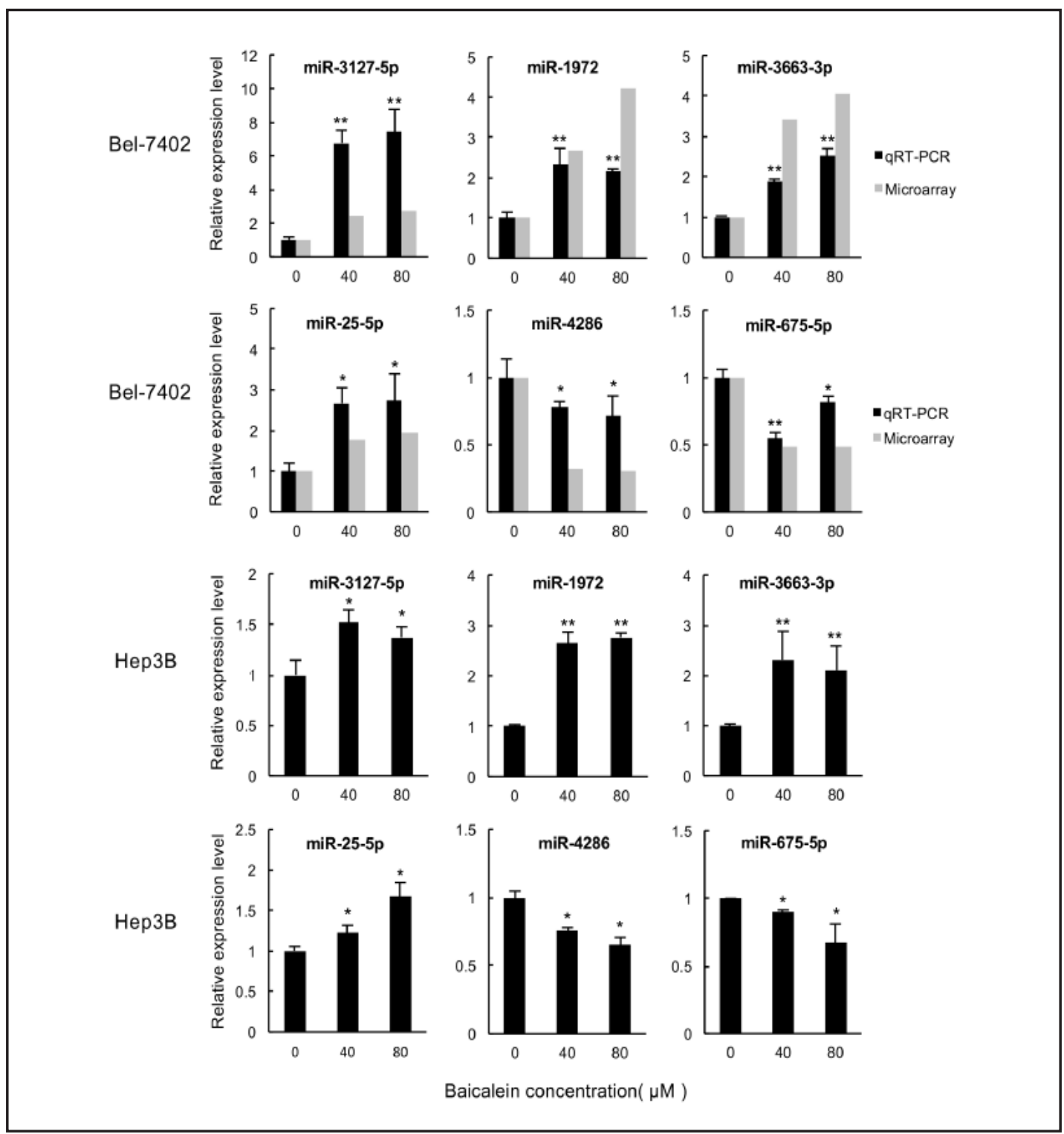

Fig. 4. Validation of miRNA microarray results by qRT-PCR in Bel-7402 and Hep3B cells. Hep3B or Bel-7402 cells were incubated with baicalein $(0,40$ and $80 \mu \mathrm{M})$ for $24 \mathrm{~h}$, and the miRNA expression level was detected by qRT-PCR and normalized to expression levels of U6. Data represent the mean \pm SD from three independent experiments. ${ }^{*} p<0.05 ;{ }^{* *} p<0.01$.

that overexpression of miR-3127-5p induced the $S$ phase arrest in Bel-7402 cells (Fig. 6B and C) and found both mRNA and protein level of p21 and p27 are able to be up-regulated by miR-3127-5p overexpression (Fig. 6D, E and F). We also found that the phosphorylation level of AKT at both Thr308 and Ser473 were significantly reduced by overexpression of miR-3127-5p (Fig. 6G and H). Furthermore, we examined the expression level of miR3127-5p in human normal hepatocyte cell line L-O2 and seven human HCC cell lines (Huh7, SMMC-7721, Hep3B, HepG2, Bel-7402, Bel-7404 and MHCC97H) using qRT-PCR and the result showed that miR-3127-5p exhibited a significantly low expression in HCC cell lines compared to normal liver cell line L-02 (Fig. 6I). These results indicate that the miR-3127-5p play a tumor-suppressive role in HCC cells. 


\section{Cellular Physiology Cell Physiol Biochem 2017;41:1519-1531

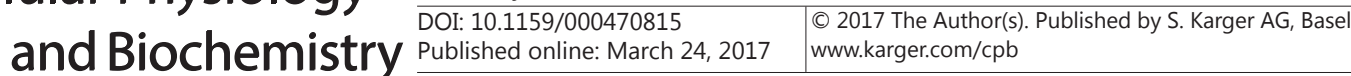 \\ Bie et al.: Baicalein Alters miRNA Expression Profiles in Bel-7402 Liver Cancer Cells}

Fig. 5. Representative results of GO and KEGG pathway enrichment for predicted miRNA target genes. (A) Representative GO terms; (B) Representative KEGG pathway terms. FDR value $<0.01$ for all significant GO and pathway terms.

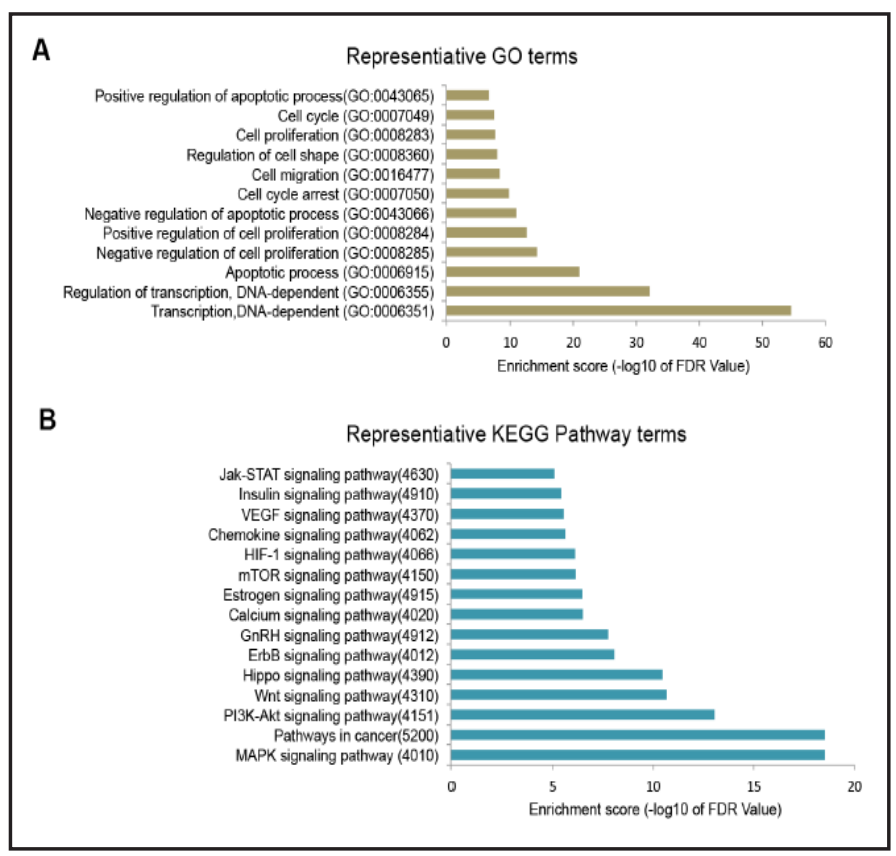

\section{Discussion}

In recent years, Chinese herb-derived compounds have attracted more and more attention due to their anti-cancer functions. Baicalein, a nature herbal product, has been demonstrated to possess significant anti-cancer effect in various types of cancer, including HCC [29]. Although some cancer-related signaling molecules have been reported to participate in baicalein-mediated inhibition of proliferation, invasion and metastasis and induction of apoptosis in HCC, the molecular mechanism of baicalein on anti-hepatoma is still far from fully understood. Interestingly, emerging studies have demonstrated that some herbal product (e.g curcumin, camptothecin, andrographolide and resveratrol etc.) can perform their anti-tumor effects through affecting miRNAs expression [16, 23-25]. However, whether the antitumor effect of baicalein is related to its modulation of miRNA expression in HCC is still unknown. In this study, we profiled the changes of miRNA expression in baicaleintreated $(40$ and $80 \mu \mathrm{M})$ and untreated $(0.1 \%$ DMSO) HCC Bel-7402 cell line using microarray to determine whether baicalein alters the miRNAs expression in HCC cell.

First, we evaluated the anti-proliferative effect of baicalien on HCC Bel-7402 cell line in vitro and our result showed that baicalein could attenuate the proliferation of HCC cell line Bel-7402 by inducing cell cycle arrest at the S and G2/M phase. Previous studies have reported that baicalein could arrest the cell cycle of HCC cells at all three phase, including G0/G1, S and G2/M. For instance, Zheng et al. [4] found that baicalein could induced G0/ G1 arrest in Bel-7404 cell line; Yu et al. [30] reported that baicalein cause cell cycle arrest at $S$ phase in HepG2 and SMMC-7721 cell lines; whereas the Kuo et al. [31] study showed baicalein exerted cytotoxic effect on J5 cells resulting in G2/M arrest, these previous studies indicated that there are substantial differences in mechanism of the cell cycle arrest induced by baicalein for different HCC cell lines.

Second, we found baicalein was able to alter the miRNA expression profiles of Bel-7402 cells and 43 miRNAs were found significantly differentially expressed between baicalein treated cells and DMSO control, including some known HCC related miRNAs. For example, miR-191, an oncogenic miRNA, was shown to be highly expressed in HCC and involved in cell proliferation, apoptosis and epithelial mesenchymal transition in HCC, and its hypomethylation has been proved to be associated with poor prognosis [32], interestingly, here we found miR-191 could be down-regulated by baicalein; miR-222-5p and miR-675 were reported to be up-regulated in HCC tumor tissues and positively associated with recurrence 
A
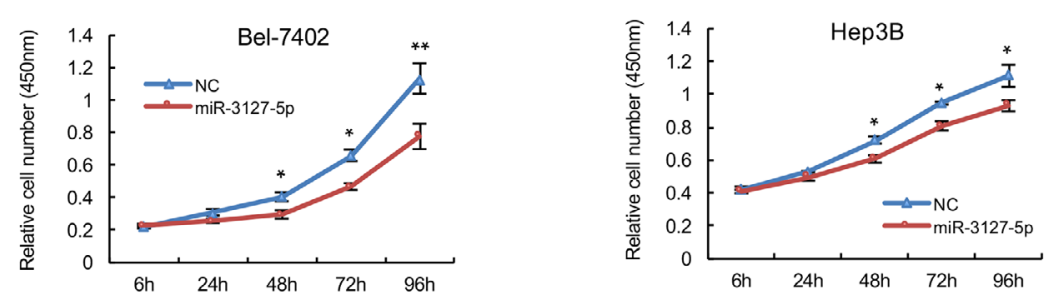

B

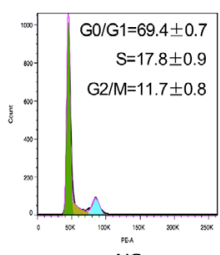

NC
C

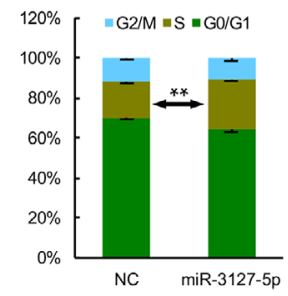

D

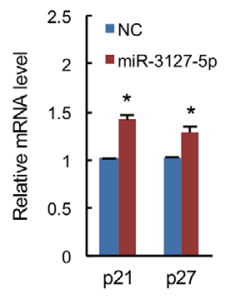

E

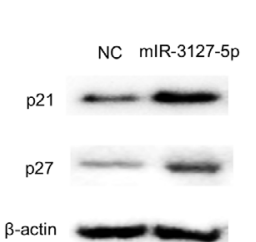

$\mathbf{F}$

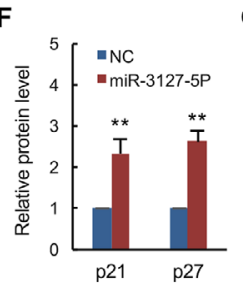

G

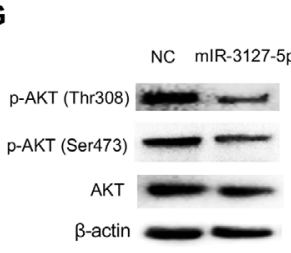

H

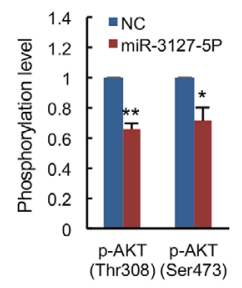

I

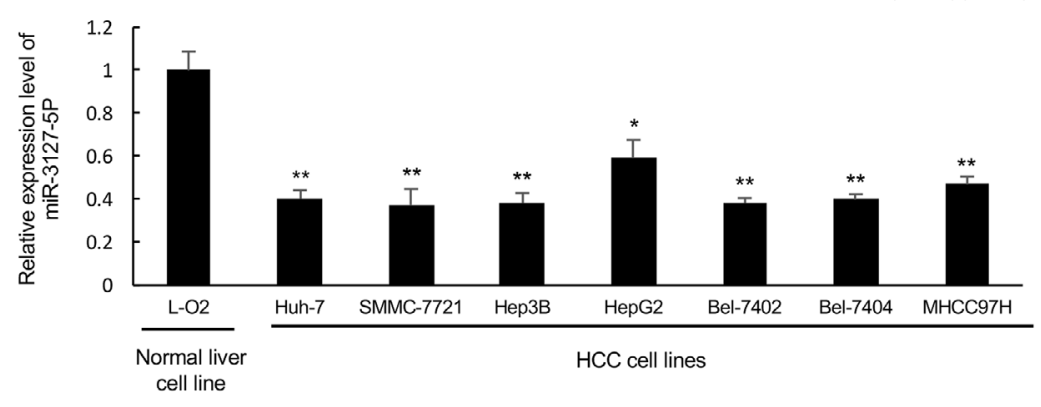

Fig. 6. MiR-3127-5p, a miRNA up-regulated by baicalein, play a tumor-suppressive role in HCC cells. (A) Analysis of cell proliferation using CCK-8 assay, Bel-7402 and Hep3B cells were transfected with miR-3127$5 \mathrm{p}$ mimics or negative control (NC) mimics; The cell cycle distribution is shown by flow cytometric histogram (B) and stacked column chart (C); Bel-7402 cells were transfected with miR-3127-5p or NC mimics or for $72 \mathrm{~h}$; mRNA level (D) and protein level (E, western bolt image; F, quantitative results) of p21 and p27 in Bel-7402 cells transfected with miR-3127-5p or NC mimics for $72 \mathrm{~h}$ was determined by qRT-PCR and western blotting, respectively; protein level (G, western bolt image; H, quantitative results) of total Akt, p-Akt (Thr 308) and p-Akt (Ser473) in Bel-7402 cells transfected with miR-3127-5p or NC mimics for $48 \mathrm{~h}$ was determined by western blotting; (I) Expression level of miR-3127-5p in normal liver cell line L-02 and seven HCC cell lines was determined by qRT-PCR. Data are shown as the mean \pm SD from three independent experiments. ${ }^{*} p<0.05,{ }^{* *} p<0.01$.

after resection [33], whereas our result showed that they were both down-regulated under baicalein treatment; miR-424-3p has been demonstrated to be down-regulated in HCC and suppresses tumor growth, cell migration and invasion through its downstream target Akt3, E2F3 and c-Myb [34, 35], nevertheless, we found miR-424-3p could be up-regulated by baicalein. In addition, previous studies have demonstrated that baicalein is able to inhibit 


\section{Cellular Physiology Cell Physiol Biochem 2017;41:1519-1531

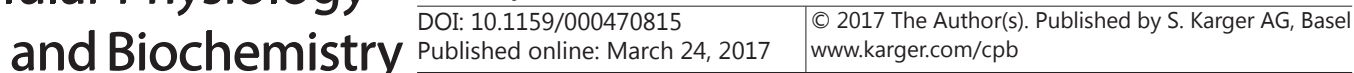 \\ Bie et al.: Baicalein Alters miRNA Expression Profiles in Bel-7402 Liver Cancer Cells}

Fig. 7. Proposed model of the mechanisms microRNA action on baicalein-induced anti-proliferation in HCC cells.

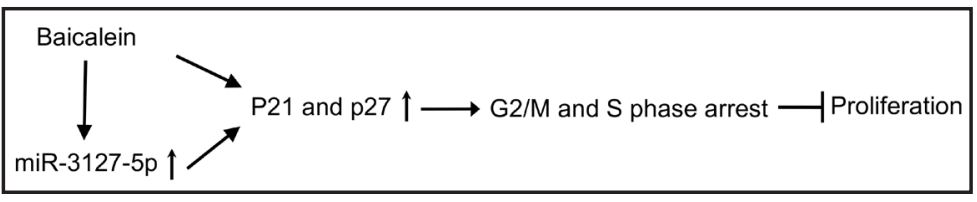

the proliferation, invasion and metastasis of HCC through inducing cell cycle arrest and apoptosis via several cancer-related signaling including MEK/ERK, JNK, mTOR, NF- $\kappa B$ etc $[5,6]$. Here, we found the putative target genes of 43 differentially expressed miRNAs were significantly enriched in GO terms of cell proliferation, cell cycle, cell cycle arrest, apoptosis process, cell migration and KEGG pathways of MAPK, PI3K-Akt, Wnt, Hippo, mTOR signaling etc, indicating our results were concordant with previous reports on the anti-hepatoma mechanisms of baicalein.

Furthermore, the qPCR results showed that changed miRNAs induced by baicalein also can be altered in Hep3B cell line similarity in Bel-7402 cells. In this study, we also found overexpression of mir-3127-5p, a down-regulated miRNA in HCC cells whereas could be upregulated by baicalein, was able to perform the anti-hepatoma effect similar to baicalein by inducing S phase arrest, up-regulating p21 and p27 expression and inactivating the PI3K/ AKT pathway. Therefore, all above results indicated that miRNA as key regulatory molecular can contribute to baicalein induced inhibition on HCC growth in vitro.

In summary, this is the first systematic study to explore the miRNA expression profiles in HCC cells with baicalein treatment on a genome-wide scale. The dynamic expression profiles, bioinformatics analysis, expression validation and biological functional characterization in vitro indicate that modulation of miRNA expression may be an important mechanism underlying the anti-hepatoma effects of baicalein (Fig. 7). This paper will provide clues and foundation for revealing the molecular mechanism of anti-hepatoma of baicalein from miRNA perspective.

\section{Acknowledgments}

This work was supported by the National Natural Science Foundation of China (No. 81503310 and 81502136) the China Postdoctoral Science Foundation funded project (No. 2015M582679), the Key Scientific and Technological Research Program for Social Development of Shaanxi Province (No. 2015SF047) and the Program for Changjiang Scholars and Innovative Research Team in University (No. IRT1171).

\section{Disclosure Statement}

The authors have no conflict of interests.

\section{References}

1 Forner A, Llovet JM, Bruix J: Hepatocellular carcinoma. Lancet 2012;379:1245-1255.

- Hu Y, Wang S, Wu X, Zhang J, Chen R, Chen M, Wang Y: Chinese herbal medicine-derived compounds for cancer therapy: a focus on hepatocellular carcinoma. J Ethnopharmacol 2013;149:601-612.

- 3 Hu B, An HM, Wang SS, Chen JJ, Xu L: Preventive and Therapeutic Effects of Chinese Herbal Compounds against Hepatocellular Carcinoma. Molecules 2016;21:142.

-4 Zheng YH, Yin LH, Grahn TH, Ye AF, Zhao YR, Zhang QY: Anticancer effects of baicalein on hepatocellular carcinoma cells. Phytother Res 2014;28:1342-1348.

-5 Chen K, Zhang S, Ji Y, Li J, An P, Ren H, Liang R, Yang J, Li Z: Baicalein inhibits the invasion and metastatic capabilities of hepatocellular carcinoma cells via down-regulation of the ERK pathway. PloS One 2013;8:e72927. 


\section{Cellular Physiology Cell Physiol Biochem 2017;41:1519-1531

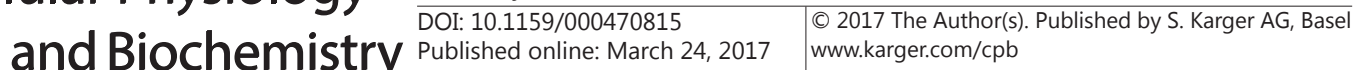

6 Wang Z, Jiang C, Chen W, Zhang G, Luo D, Cao Y, Wu J, Ding Y, Liu B: Baicalein induces apoptosis and autophagy via endoplasmic reticulum stress in hepatocellular carcinoma cells. Biomed Res Int 2014;2014:732516.

7 Liang R-R, Zhang S, Qi J-A, Wang Z-D, Li J, Liu P-J, Huang C, Le X-F, Yang J, Li Z-F: Preferential inhibition of hepatocellular carcinoma by the flavonoid Baicalein through blocking MEK-ERK signaling. Int J Oncol 2012;41:969-978.

-8 Wang YF, Li T, Tang ZH, Chang LL, Zhu H, Chen XP, Wang YT, Lu JJ: Baicalein Triggers Autophagy and Inhibits the Protein Kinase B/Mammalian Target of Rapamycin Pathway in Hepatocellular Carcinoma HepG2 Cells. Phytother Res 2015;29:674-679.

-9 Han Z, Zhu S, Han X, Wang Z, Wu S, Zheng R: Baicalein inhibits hepatocellular carcinoma cells through suppressing the expression of CD24. Int Immunopharmacol 2015;29:416-422.

-10 Xu XM, Yuan GJ, Deng JJ, Guo HT, Xiang M, Yang F, Ge W, Chen SY: Inhibition of 12-lipoxygenase reduces proliferation and induces apoptosis of hepatocellular carcinoma cells in vitro and in vivo. Hepatobiliary Pancreat Dis Int 2012;11:193-202.

-11 Chiu YW, Lin TH, Huang WS, Teng CY, Liou YS, Kuo WH, Lin WL, Huang HI, Tung JN, Huang CY, Liu JY, Wang WH, Hwang JM, Kuo HC: Baicalein inhibits the migration and invasive properties of human hepatoma cells. Toxicol Appl Pharmacol 2011;255:316-326.

12 Gao F, Sun X, Wang L, Tang S, Yan C: Downregulation of MicroRNA-145 Caused by Hepatitis B Virus X Protein Promotes Expression of CUL5 and Contributes to Pathogenesis of Hepatitis B Virus-Associated Hepatocellular Carcinoma. Cell Physiol Biochem 2015;37:1547-1559.

13 Sun JJ, Chen GY, Xie ZT: MicroRNA-361-5p Inhibits Cancer Cell Growth by Targeting CXCR6 in Hepatocellular Carcinoma. Cell Physiol Biochem 2016;38:777-785.

14 Hayes J, Peruzzi PP, Lawler S: MicroRNAs in cancer: biomarkers, functions and therapy. Trends Mol Med 2014;20:460-469.

15 Hong M, Wang N, Tan HY, Tsao SW, Feng Y: MicroRNAs and Chinese Medicinal Herbs: New Possibilities in Cancer Therapy. Cancers 2015;7:1643-1657.

16 Sreenivasan S, Thirumalai K, Danda R, Krishnakumar S: Effect of curcumin on miRNA expression in human Y79 retinoblastoma cells. Curr Eye Res 2012;37:421-428.

17 Sun M, Estrov Z, Ji Y, Coombes KR, Harris DH, Kurzrock R: Curcumin (diferuloylmethane) alters the expression profiles of microRNAs in human pancreatic cancer cells. Mol Cancer Ther 2008;7:464-473.

18 Zhang J, Du Y, Wu C, Ren X, Ti X, Shi J, Zhao F, Yin H: Curcumin promotes apoptosis in human lung adenocarcinoma cells through miR-186* signaling pathway. Oncol Rep 2010;24:1217-1223.

19 Yang J, Cao Y, Sun J, Zhang Y: Curcumin reduces the expression of Bcl-2 by upregulating miR-15a and miR16 in MCF-7 cells. Med Oncol 2010;27:1114-1118.

20 Zhang W, Bai W, Zhang W: MiR-21 suppresses the anticancer activities of curcumin by targeting PTEN gene in human non-small cell lung cancer A549 cells. Clin Transl Oncol 2014;16:708-713.

21 Guo H, Xu Y, Fu Q: Curcumin inhibits growth of prostate carcinoma via miR-208-mediated CDKN1A activation. Tumour Biol 2015;36:8511-8517.

-22 Zeng CW, Zhang XJ, Lin KY, Ye H, Feng SY, Zhang H, Chen YQ: Camptothecin induces apoptosis in cancer cells via microRNA-125b-mediated mitochondrial pathways. Mol Pharmacol 2012;81:578-586.

-23 Huang N, Wu J, Qiu W, Lyu Q He J, Xie W, Xu N, Zhang Y: MiR-15a and miR-16 induce autophagy and enhance chemosensitivity of Camptothecin. Cancer Biol Ther 2015;16:941-948.

24 Lu B, Sheng Y, Zhang J, Zheng Z, Ji L: The altered microRNA profile in andrographolide-induced inhibition of hepatoma tumor growth. Gene 2016;588:124-133.

25 Venkatadri R, Muni T, Iyer AK, Yakisich JS, Azad N: Role of apoptosis-related miRNAs in resveratrol-induced breast cancer cell death. Cell Death Dis 2016;7:e2104.

26 Rui XJ, Yan X, Zhang K: Baicalein inhibits the migration and invasion of colorectal cancer cells via suppression of the AKT signaling pathway. Oncol Lett 2016;11:685-688.

27 Zhang HB, Lu P, Guo QY, Zhang ZH, Meng XY: Baicalein induces apoptosis in esophageal squamous cell carcinoma cells through modulation of the PI3K/Akt pathway. Oncol Lett 2013; 11:685-688.

28 Guo Z, Hu X, Xing Z, Xing R, Lv R, Cheng X, Su J, Zhou Z, Xu Z, Nilsson S: Baicalein inhibits prostate cancer cell growth and metastasis via the caveolin-1/AKT/mTOR pathway. Mol Cell Biochem 2015;406:111-119.

29 Liu H, Dong Y, Gao Y, Du Z, Wang Y, Cheng P, Chen A, Huang H: The Fascinating Effects of Baicalein on Cancer: A Review. Int J Mol Sci 2016;17:1681 


\section{Cellular Physiology Cell Physiol Biochem 2017;41:1519-1531

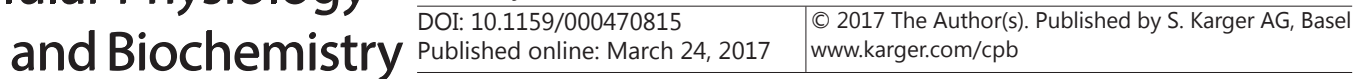 \\ Bie et al.: Baicalein Alters miRNA Expression Profiles in Bel-7402 Liver Cancer Cells}

30 Yu Y, Pei M, Li L: Baicalin induces apoptosis in hepatic cancer cells in vitro and suppresses tumor growth in vivo. Int J Clin Exp Med 2015;8:8958-8967.

-31 Kuo HM, Tsai HC, Lin YL, Yang JS, Huang AC, Yang MD, Hsu SC, Chung MC, Gibson Wood W, Chung JG: Mitochondrial-dependent caspase activation pathway is involved in baicalein-induced apoptosis in human hepatoma J5 cells. Int J Oncol 2009;35:717-724.

-32 Nagpal N, Kulshreshtha R: miR-191: an emerging player in disease biology. Front Genet 2014;5:99.

33 Sato F, Hatano E, Kitamura K, Myomoto A, Fujiwara T, Takizawa S, Tsuchiya S, Tsujimoto G, Uemoto S, Shimizu K: MicroRNA profile predicts recurrence after resection in patients with hepatocellular carcinoma within the Milan Criteria. PloS One 2011;6:e16435.

-34 Yang H, Zheng W, Shuai X, Chang RM, Yu L, Fang F, Yang LY: MicroRNA-424 inhibits Akt3/E2F3 axis and tumor growth in hepatocellular carcinoma. Oncotarget 2015;6:27736-27750.

35 Yu L, Ding GF, He C, Sun L, Jiang Y, Zhu L: MicroRNA-424 is down-regulated in hepatocellular carcinoma and suppresses cell migration and invasion through c-Myb. PloS One 2014;9:e91661. 